\title{
Kartografie des Herzens mittels Mappingsystemen
}

Gerade in der Elektrophysiologie, einer Subdisziplin der Kardiologie, hat mit der Ankunft des elektroanatomischen Mappings in den vergangenen Jahren ein Quantensprung stattgefunden, und dies in vielerlei Hinsicht. Die Initialzündung für diese Entwicklung entstammt den Arbeiten von Shlomo Ben-Haim [1].

Mit Mapping ist in der Elektrophysiologie das Kartografieren des Herzens gemeint. Es werden mit Mappingsystemen, die im Artikel von Kany und Saguner [2] im Detail vorgestellt werden, dreidimensionale Karten der «chamber of interest» erstellt, das heisst, derjenigen Herzkammer, von der eine zu behandelnde Arrhythmie mutmasslich ausgeht. Da anhand des impedanzbasierten und/oder elektromagnetisch basierten Systems die Katheterelektrode, sprich die Spitze des Katheters, eindeutig räumlich Koordinaten zugeordnet werden kann, ist es möglich, die anatomischen Verhältnisse mit hoher Genauigkeit (ca. $1 \mathrm{~mm}$ ) abzubilden. Während diese Eigenschaft den zweiten Wortanteil des Begriffs «elektroanatomisch» meint, beschreibt der erste Teil die Möglichkeit, die elektrischen Signale in der Herzkammer aufzuzeichnen, eigentlich analog einem Elektrokardiogramm (EKG), das allerdings direkt im Herzen und nicht an der Körperoberfläche abgeleitet wird. Durch das Zusammensetzen der elektrischen und der anatomischen Information und die dreidimensionale Darstellung mit Farbcodierung der zeitlichen oder lokalen Spannungsinformation können der elektrische Erregungsablauf in der untersuchten Herzkammer sowie interessante Narbenareale für den Elektrophysiologen sichtbar gemacht werden. Die Analyse der intrakardialen Elektrogramme sowie die Antwort auf Stimulationsmanöver ergeben dann ein Gesamtbild, das in den allermeisten Fällen eine eindeutige Lokalisation des Ursprungs der Arrhythmie und deren Verödung (Ablation) zulässt.

Die Autoren des Manuskripts zeigen in ihrer Arbeit schön auf, wie hilfreich diese oben beschriebenen elektroanatomischen Mappingsysteme in der modernen Elektrophysiologie sind. Dabei machen sie deutlich, dass durch deren Einsatz gerade die Pulmonalvenenisolation bei Vorhofflimmern, der häufigsten Rhythmusstörung im klinischen Alltag, mit grosser Sicherheit und praktisch ohne Einsatz von Röntgenstrahlung erfolgen kann. So ist die Pulmonalvenenisolation heute zum absoluten Routineeingriff und die am häufigsten durchgeführte Katheterablation geworden.

Die Entwicklung der elektroanatomischen Mappingsysteme ging parallel zur Entdeckung, dass Vorhofflimmern in vielen Fällen durch ektope Aktivität in den Pulmonalvenen ausgelöst wird. Die Katheterablation von Vorhof- flimmern war damit geboren und eignete sich vorzüglich, um die gerade erfundenen Mappingsysteme weiter voranzubringen. Somit sind die Mappingsysteme eben auch ein hervorragendes Beispiel für medizinischen Fortschritt. Während die Mappingsysteme für die Behandlung einer häufigen Krankheit (Vorhofflimmern) weiterentwickelt worden waren, wurde plötzlich erkannt, dass diese auch in anderen Situationen (z.B. Patienten mit komplexen Arrhythmien nach Herzinfarkt oder bei kongenitalen Herzerkrankungen) sehr erfolgreich eingesetzt werden können. Für diese vergleichsweise seltenen Arrhythmien wären jedoch die Mappingsysteme wahrscheinlich nie primär entwickelt worden.

Während konventionelle Katheterablationen wie die Ablation von supraventrikulären Tachykardien problemlos ohne Mappingsystem durchgeführt werden, sind die elektroanatomischen Mappingsysteme bei der Behandlung von Patienten bei komplexen Herzrhythmusstörungen (Kammertachykardien, Vorhofflimmern, angeborene Herzfehler) nicht mehr aus dem klinischen Alltag wegzudenken. Es wäre wie eine Wanderung, bei der man die Landkarte mit dem darauf eingezeichneten Ziel nicht zur Hand hätte.

Gerade in Zeiten, in denen die grundsätzliche Vorgabe lautet, Kosten zu reduzieren, ist es sehr wichtig, gegenüber den Prämienzahlern, den Versicherern, den politischen Organen, aber auch gegenüber den Praktikern als Bindeglied zwischen Patient und Spezialist, die «Nutzenexplosion» in der Medizin der Kostenexplosion gegenüberzustellen. Die technologischen Fortschritte beim Mapping in der Elektrophysiologie sind hierfür ein exzellentes Beispiel.

\section{Bibliografie}

1. Shlomo A, Ben-Haim SA, Osadchy D, Scnuster I, Lior Gepstein L, Hayam G, Josephson ME: Nonfluoroscopic, in vivo navigation and mapping technology. Nature Med 1996; 2:1393-1395.

2. Shinwan Kany S, Saguner AM: Die Rolle des elektroanatomischen Mappings in der Rhythmologie. Praxis 2018; 107: $1326-1332$

Prof. Michael Kühne

Leitender Arzt Kardiologie/Elektrophysiologie

Universitätsspital Basel

Petersgraben 4

4031 Basel

michael.kuehne@usb.ch 